\title{
Heparin in the treatment of aneurysmal subarachnoid hemorrhage: a systematic review and meta-analysis
}

\author{
Patrick P. Lukito, MD, ${ }^{1}$ Hendry Lie, MD, ${ }^{1}$ Karina Helsa, MD, ${ }^{1}$ and Julius July, MD, PhD² \\ ${ }^{1}$ Faculty of Medicine and ${ }^{2}$ Department of Neurosurgery, Neuroscience Centre Siloam Hospital, Faculty of Medicine, Universitas \\ Pelita Harapan, Tangerang, Banten, Indonesia
}

\begin{abstract}
OBJECTIVE Cerebral vasospasm and the resulting infarction remain the most devastating complications of aneurysmal subarachnoid hemorrhage (aSAH). Limited treatment options are available, with nimodipine as the only approved prophylactic medication. In addition to its anticoagulant properties, heparin also has a pleiotropic and anti-inflammatory effect that could be beneficial in vasospasm. In this study, the authors sought to evaluate the efficacy and safety of heparin in the treatment of aSAH.
\end{abstract}

METHODS The PubMed, EBSCOhost, Europe PMC, and Cochrane Central databases were searched to find studies including patients with aSAH who were treated with intravenous unfractionated heparin (UFH) after an aneurysm-securing procedure. Studies that did not include a comparison with UFH or low-molecular-weight heparin in deep vein thrombosis prophylactic doses were excluded. The primary outcome was cerebral vasospasm, and the secondary outcomes were cerebral infarction, clinical deterioration caused by delayed cerebral ischemia, bleeding complications, and thromboembolism complications.

RESULTS Overall, 5 nonrandomized studies were included; 4 studies evaluated the safety and 3 studies evaluated the efficacy of intravenous heparin. From the analysis of 3 studies with a total of 895 patients, administration of intravenous UFH for $>48$ hours was related to a significantly lower rate of cerebral infarction (OR $0.44,95 \% \mathrm{Cl} 0.25-0.79$ ). No significant association was found with other efficacy outcomes. Regarding cognitive outcome, one study found a significant improvement in Montreal Cognitive Assessment scores; however, the functional outcome as indicated by the modified Rankin Scale score was not improved by heparin administration. From the analysis of 4 studies with 1099 patients, no significant increases in bleeding and other complications were found.

CONCLUSIONS Administration of intravenous UFH for more than 48 hours reduced the rate of cerebral infarction with a good safety profile. This result supports the ongoing clinical trial.

Systematic review registration no.: CRD42021254654 (https://www.crd.york.ac.uk/prospero/)

https://thejns.org/doi/abs/10.3171/2021.12.FOCUS21419

KEYWORDS aneurysmal subarachnoid hemorrhage; heparin; treatment; efficacy; safety; outcome

$\mathrm{C}$ EREBRAL vasospasm and the resulting infarction are the leading contributors to mortality and morbidity in patients with aneurysmal subarachnoid hemorrhage (aSAH). ${ }^{1,2}$ Cerebral infarction occurs through a multifactorial process, and studies point to vasospasm, microthromboembolism, microvascular constriction, neuroinflammation, blood-brain barrier disruption, cortical spreading ischemia, and impaired cerebral autoregulation. ${ }^{3-5}$ Despite the high mortality and morbidity rate associated with aSAH, there are few prophylactic treatment options. Nimodipine is the only prophylactic treatment approved for SAH that it has been shown to improve outcome, ${ }^{6,7}$ while several other trials with clazosentan, statins, and anti-inflammatory drugs have been disappointing. ${ }^{8-10}$

Heparin is widely used for prophylaxis and treatment of thromboembolisms for its anticoagulant properties. Heparin also has a pleiotropic effect, with various related beneficial effects. It acts as an anti-inflammatory agent, as it alters vasomotor regulations, and recent studies have suggested its use as a potential therapeutic inhibitor of in-

ABBREVIATIONS aPTT = activated partial thromboplastin time; aSAH = aneurysmal subarachnoid hemorrhage; $\mathrm{DCl}=$ delayed cerebral ischemia; $\mathrm{DVT}=$ deep vein thrombosis; ET-1 = endothelin-1; GRADE = Grading of Recommendations Assessment, Development and Evaluation; HIT = heparin-induced thrombocytopenia; LDIVH = low-dose intravenous heparin; LMWH = low-molecular-weight heparin; MoCA = Montreal Cognitive Assessment; mRS = modified Rankin Scale; OR = odds ratio; ROBINS-I = Risk Of Bias In Nonrandomized Studies-of Interventions; UFH = unfractionated heparin; WFNS = World Federation of Neurosurgical Societies.

SUBMITTED July 23, 2021. ACCEPTED December 10, 2021.

INCLUDE WHEN CITING DOI: 10.3171/2021.12.FOCUS21419. 
flammation, which are all the relevant mechanisms for the treatment of aSAH-induced brain injury. ${ }^{11,12}$ Preclinical investigations in rodent models found that heparin reduced edema, neuroinflammation, demyelination, and transsynaptic neuronal apoptosis. ${ }^{13}$ Because of the promising potential of heparin, we aimed to evaluate the latest evidence on the safety and efficacy of its usage in treating aSAH.

\section{Methods \\ Database and Literature Search}

A literature search was performed using PubMed, EBSCOhost, Europe PMC, and Cochrane Central from database inception until June 15, 2021, with the following keywords: ["subarachnoid hemorrhage" or "SAH" or "aSAH" and "heparin"]. The references of relevant studies were also reviewed to supplement the search. Only English-language literature was included. Two authors (P.P.L. and H.L.) independently performed the initial search and screened the title and abstract of relevant studies. Any discrepancies were resolved by discussion with a third author (J.J.).

\section{Study Selection}

The criteria for selecting the study were as follows: 1) types of studies: randomized, nonrandomized, prospective, and retrospective studies; 2) types of participants: patients with acute aSAH that had been secured by either clipping or coiling; 3) types of intervention: intravenous unfractionated heparin (UFH) in a therapeutic dose; 4) types of comparison: comparison with UFH or low-molecular-weight heparin (LMWH) in deep vein thrombosis (DVT) prophylactic doses; and 5) the primary outcome of angiographic vasospasm, with secondary outcomes, including cerebral infarction, clinical deterioration caused by delayed cerebral ischemia (DCI), bleeding complications, and thromboembolism complications. Studies without comparison to heparin in prophylactic doses were excluded. Review articles, editorials, correspondences, case reports, case series, and non-English-language articles were also excluded.

\section{Data Extraction}

Two independent authors (P.P.L. and H.L.) performed data extraction for this analysis using a standardized form that included the authors, publication year, study design, patient characteristics, and outcomes measured. Extracted data were then compared, with any discrepancies resolved by discussion.

We used the definitions suggested by Vergouwen et al. ${ }^{14}$ Angiographic vasospasm was defined as narrowing of a large cerebral artery in any vascular territory evidenced on angiographic vasospasm from CTA, MRA, or DSA studies. Cerebral infarction was defined as the presence of cerebral infarction on CT or MRI of the brain within 6 weeks after aSAH, or on the latest CT or MRI study obtained before death within 6 weeks, or proven at autopsy but not present on CT or MRI study between 24 and 48 hours after early aneurysm occlusion and not attributable to other causes such as surgical clipping or endovascular treatment. Clinical deterioration caused by DCI was defined as the occurrence of focal neurological impairment or a decrease of at least 2 points on the Glasgow Coma Scale. This deterioration should last for at least 1 hour, is not apparent immediately after aneurysm occlusion, and cannot be attributed to other causes by means of clinical assessment, CT or MRI studies of the brain, and appropriate laboratory studies. However, since not all studies followed these suggested definitions, other definitions were allowed, provided that they did not differ significantly. Safety outcomes consisted of bleeding and clotting complications. A bleeding complication was defined as any bleeding complications after administration of intravenous UFH, and a clotting complication was defined as DVT and heparin-induced thrombocytopenia (HIT). Aside from the outcome, we also collected data on study design, patient baseline characteristics, and the intervention given. The data were then entered in Review Manager version 5 (The Cochrane Collaboration) software by one author (P.P.L.) and double-checked by another author (H.L.). A two-bytwo contingency table was constructed for each outcome.

\section{Statistical Analysis}

Due to the expected clinical heterogeneity between studies, a random-effects model (DerSimonian and Laird) was used regardless of statistical heterogeneity. Outcomes were compared between therapeutic and prophylactic doses of heparin using odds ratios and 95\% confidence intervals. Statistical significance was set at $p<0.05$. The results are presented as forest plot diagrams. Heterogeneity across studies was evaluated using the inconsistency index $\left(\mathrm{I}^{2}\right)$ test. Risk of bias was assessed by one author (P.P.L.) in the study level with the Cochrane risk-of-bias tool (The Cochrane Collaboration) for randomized studies and the Risk Of Bias In Nonrandomized Studies-of Interventions (ROBINS-I) tool for nonrandomized studies. A regressionbased Egger's test was performed to evaluate the risk of publication bias. Certainty of evidence was assessed using the Grading of Recommendations Assessment, Development and Evaluation (GRADE; https://www.gradeworking group.org) approach. Analysis was performed with Review Manager version 5.3 (The Cochrane Collaboration).

During the review process, we found that administration of intravenous UFH for $>48$ hours was related to better efficacy. We, therefore, conducted a sensitivity analysis with patients who received intravenous UFH for $>48$ hours. This study was conducted following the 2015 PRISMA guidelines for a systematic review. This review has been registered at the international prospective register of systematic reviews (PROSPERO) under the registration number CRD42021254654 (https://www.crd.york. ac.uk/prospero/).

\section{Results}

A total of 469 studies were retrieved from PubMed, EBSCOhost, Europe PMC, and Cochrane Central; $158 \mathrm{du}-$ plicate studies were excluded, and the remaining 311 studies were screened. From the initial screening, 23 full-text articles were evaluated for eligibility. From the 23 articles evaluated, 18 were excluded because of the lack of available data on the desired outcome, leaving 5 studies for the final analysis (Fig. 1). ${ }^{15-19}$ 


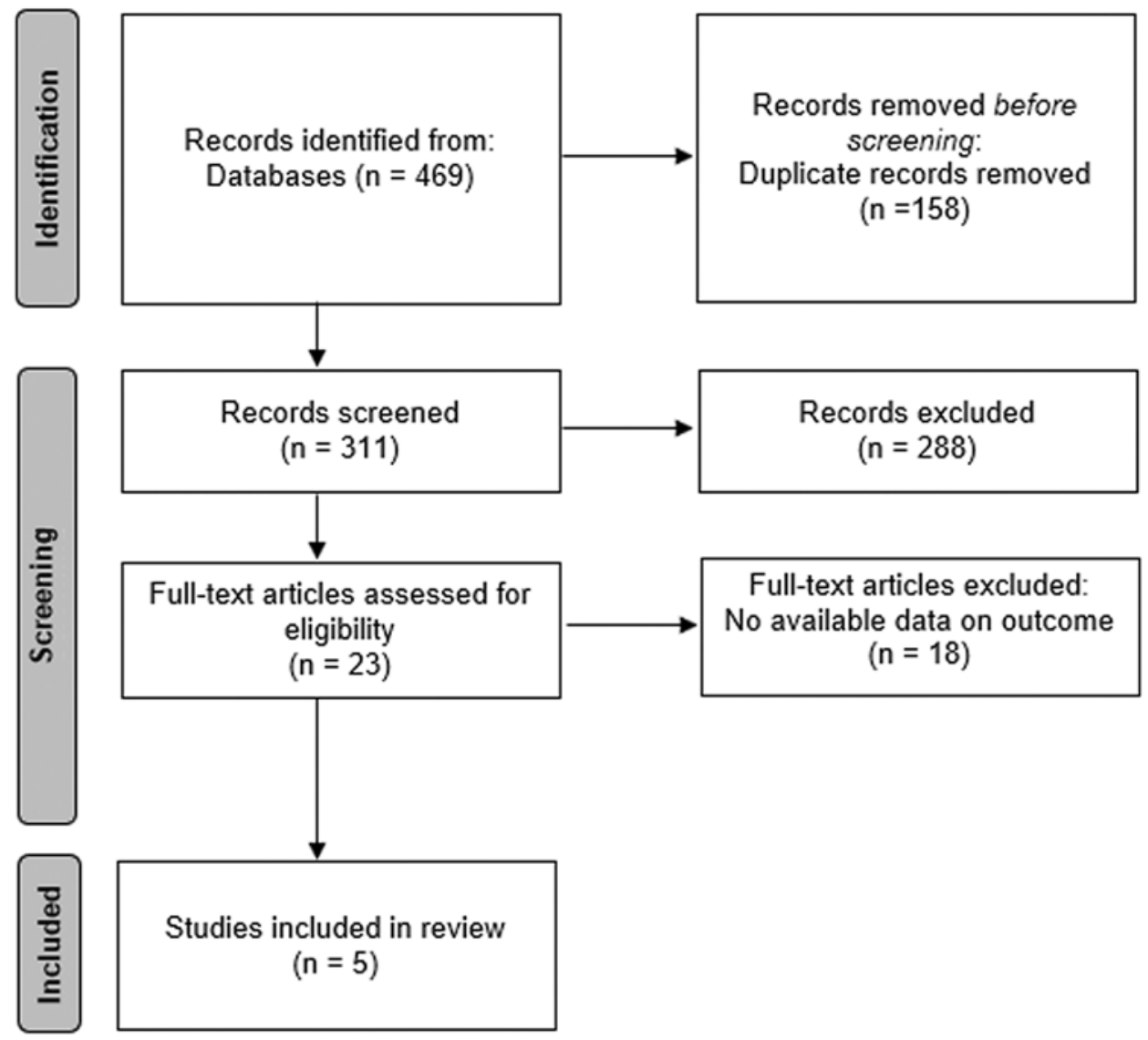

FIG. 1. Study flow diagram. Data added to the PRISMA template [from Page MJ, McKenzie JE, Bossuyt PM, Boutron I, Hoffmann TC, Mulrow CD, et al. The PRISMA 2020 statement: an updated guideline for reporting systematic reviews. BMJ 2021;372:n71. doi: 10.1136/bmj.n71].

\section{Study Characteristics}

The characteristics of the included studies are presented in Table 1. All were retrospective cohort studies except the study by Bruder et al., which was a case series study with matched-pair analysis using multivariate and propensity score matching to select patients for the control group. ${ }^{16}$ Simard et al., James et al., and Kole et al. administered the Maryland low-dose intravenous heparin (LDIVH) protocol in the experimental group. ${ }^{15,18,19}$ Bruder et al. and Kunz et al. administered intravenous heparin using activated partial thromboplastin time (aPTT) as a marker. ${ }^{16,17}$ Bruder et al. administered the heparin for up to 7 days and divided the experimental group into those receiving intravenous UFH for $<$ or $>48$ hours, while Kunz et al. did not specify a time frame. In the control group, 4 studies administered subcutaneous UFH or LMWH in prophylaxis dose, , $^{15,16,18,19}$ while 1 study administered intravenous UFH. ${ }^{17}$ Baseline characteristics of patients in all but one study, that of Kole et al., ${ }^{19}$ were not statistically different. In their study, the intervention group had worse World Federation of Neurosurgical Societies (WFNS) and Fisher grades.

Since all the included studies were not randomized, we used the ROBINS-I tool to assess bias (Table 2). Overall, 4 studies were assessed as having a moderate risk of bias, while 1 study had a serious risk of bias. Due to the nature of the studies, confounding bias was prevalent, especially in the study by Kole et al., ${ }^{19}$ in which their intervention group had significantly worse WFNS and Fisher grades. Publication bias was not evaluated, as there was an inadequate number of studies to use a regression-based assessment properly.

\section{Angiographic Vasospasm}

Three studies assessed angiographic vasospasm after an aneurysm-securing procedure. The definitions used in these studies complied with the definition suggested by Vergouwen et al. ${ }^{14}$ These studies included 473 patients in the therapeutic group and 563 in the prophylactic group. Overall, there was a higher rate of angiographic vasospasm in the therapeutic group, although the difference was not significant with an odds ratio (OR) of 1.29 (95\% CI $0.46-3.59, \mathrm{p}=0.63 ; \mathrm{I}^{2}=93 \%, \mathrm{p}<0.00001$ ) (Fig. 2A). Two of those studies evaluated the severity of vasospasm. In both studies, severe vasospasm was defined as $>66 \%$ vessel narrowing, as seen on angiography. The rate of severe angiographic vasospasm was higher in the prophylactic group, although it failed to reach statistical significance with an OR of 0.88 (95\% CI 0.28-2.74, p = 0.82; $\mathrm{I}^{2}=91 \%$, $\mathrm{p}=0.0009)($ Fig. 2B). 


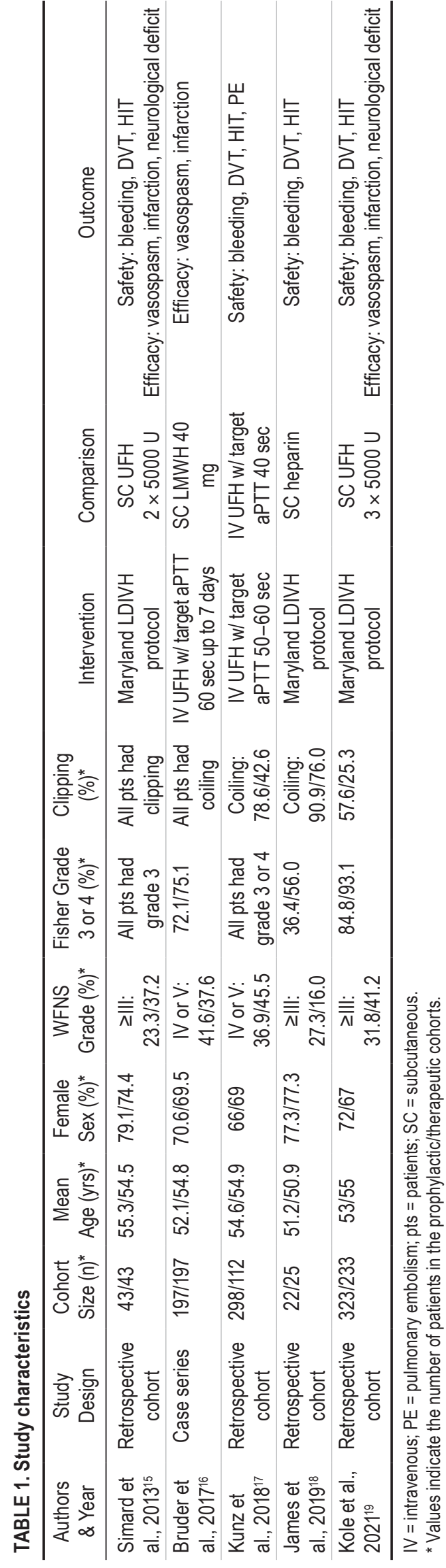

\section{Cerebral Infarction}

Three studies assessed cerebral infarction. The definition used in all 3 studies differed from the one suggested by Vergouwen et al. ${ }^{14}$ They did not describe whether the infarction occurred within the suggested criteria of 6 weeks. They also did not mention excluding infarction that occurred within 24 to 48 hours after an aneurysm-securing procedure. However, those studies excluded hypodensities related to other causes such as surgical procedures, ventricular catheter, or intraparenchymal hematoma. These studies included 473 patients in the therapeutic group and 563 patients in the prophylactic group. Overall, the rate is lower in the therapeutic group; however, it still did not reach statistical significance with an OR of 0.53 (95\% CI $0.26-1.07, \mathrm{p}=0.08 ; \mathrm{I}^{2}=65 \%, \mathrm{p}=0.06$ ) (Fig. $2 \mathrm{C}$ ).

\section{Clinical Deterioration Caused by DCI}

Two studies assessed clinical deterioration caused by DCI. Simard et al. presented the number of patients who experienced a new neurological deterioration after the aneurysm-securing procedure. ${ }^{15}$ The authors did not specify the criteria, onset, or duration of the impairment. They attributed the impairment to vasospasm, although they did not describe any measures to exclude other causes. On the other hand, Kole et al. used the term "delayed neurological deficit," which was defined as new neurological decline compared with baseline after the aneurysm-securing procedure, including changes in mental status, pronator drift, or other focal neurological deficit in the absence of hydrocephalus, mass lesion, surgical or endovascular injury, or another adjudicated medical cause. ${ }^{19}$ The authors did not describe their methods of evaluating mental status and did not mention the time frame. These studies included 276 patients and 366 patients in the therapeutic and prophylactic groups, respectively. The rate was lower in the therapeutic group, but it failed to reach statistical significance with an OR of $0.31\left(95 \%\right.$ CI $0.06-1.77, \mathrm{p}=0.19 ; \mathrm{I}^{2}=87 \%$, $\mathrm{p}=0.006$ ) (Fig. 2D). Simard et al. followed patients for 6 weeks and found that none of the patients in the therapeutic group experienced persistent neurological deficits compared with 5 patients in the prophylactic group.

\section{Bleeding Complications}

Four papers assessed bleeding complications that might arise from a higher dose of heparin. These complications included intracerebral hemorrhage, epidural and subdural hematoma, and external ventricular drain track hemorrhage. All complications were radiologically assessed. These studies included 413 patients with therapeutic doses and 686 patients with prophylactic doses. The therapeutic group had a higher rate of bleeding complications, although the difference was not significant; the pooled OR was 1.25 (95\% CI 0.60-2.60, $\mathrm{p}=0.56 ; \mathrm{I}^{2}=44 \%, \mathrm{p}=0.17$ ) (Fig. 3A).

\section{Deep Vein Thrombosis}

Two studies assessed DVT occurrence in their patients. Kole et al. and Kunz et al. used duplex examination..$^{17,19}$ These studies included 345 patients in the therapeutic group and 621 in the prophylactic group. The rate of DVT was lower in the therapeutic group, but it did not reach sta- 
TABLE 2. Risk-of-bias assessment using the ROBINS-I tool

\begin{tabular}{|c|c|c|c|c|c|c|c|c|}
\hline $\begin{array}{l}\text { Authors } \\
\text { \& Year }\end{array}$ & Confounding & Selection & $\begin{array}{l}\text { Intervention } \\
\text { Classification }\end{array}$ & $\begin{array}{l}\text { Deviation From } \\
\text { Intervention }\end{array}$ & $\begin{array}{l}\text { Missing } \\
\text { Data }\end{array}$ & $\begin{array}{l}\text { Measurement of } \\
\text { Outcome }\end{array}$ & $\begin{array}{c}\text { Selection of } \\
\text { Reported Results }\end{array}$ & Overall \\
\hline $\begin{array}{l}\text { Simard et al., } \\
2013^{15}\end{array}$ & Moderate & Low & Moderate & Low & Low & Low & Moderate & Moderate \\
\hline $\begin{array}{l}\text { Bruder et al., } \\
2017^{16}\end{array}$ & Low & Low & Moderate & Low & Low & Low & Moderate & Moderate \\
\hline $\begin{array}{l}\text { Kunz et al., } \\
2018^{17}\end{array}$ & Moderate & Low & Moderate & Low & Low & Moderate & Moderate & Moderate \\
\hline $\begin{array}{l}\text { James et al., } \\
2019^{18}\end{array}$ & Moderate & Low & Moderate & Low & Low & Low & Moderate & Moderate \\
\hline $\begin{array}{l}\text { Kole et al., } \\
2021^{19}\end{array}$ & Serious & Low & Moderate & Low & Low & Moderate & Moderate & Serious \\
\hline
\end{tabular}

\section{A}

\begin{tabular}{|c|c|c|c|c|c|c|}
\hline \multirow[b]{2}{*}{ Study or Subgroup } & & & & \multirow[b]{2}{*}{ Weight } & \multirow[b]{2}{*}{ M-H, Random, $95 \% \mathrm{Cl}$} \\
\hline & Events & Total & Events & Total & & \\
\hline Bruder $2017^{16}$ & 79 & 197 & 93 & 197 & $35.1 \%$ & $0.75[0.50,1.12]$ \\
\hline Kole $2021^{19}$ & 151 & 233 & 124 & 323 & $35.5 \%$ & $2.96[2.08,4.19]$ \\
\hline Simard $2013^{15}$ & 25 & 43 & 26 & 43 & $29.4 \%$ & $0.91[0.38,2.15]$ \\
\hline Total $(95 \% \mathrm{Cl})$ & & 473 & & 563 & $100.0 \%$ & $1.29[0.46,3.59]$ \\
\hline Total events & 255 & & 243 & & & \\
\hline Heterogeneity: Tau ${ }^{2}$ & 74; $\mathrm{Chi}^{2}=$ & $29, \mathrm{df}=$ & $2(P<0.0$ & 1); $\left.\right|^{2}=$ & $93 \%$ & \\
\hline
\end{tabular}

B

\begin{tabular}{|c|c|c|c|c|c|}
\hline \multirow[b]{2}{*}{ Study or Subgroup } & \multicolumn{2}{|c|}{ Therapeutic dose } & \multicolumn{2}{|c|}{ Prophylactic dose } & \multirow[b]{2}{*}{ Weight } \\
\hline & Events & Total & Events & Total & \\
\hline Bruder $2017^{16}$ & 28 & 197 & 50 & 197 & $49.5 \%$ \\
\hline Kole $2021^{19}$ & 45 & 233 & 43 & 323 & $50.5 \%$ \\
\hline Total (95\% Cl) & & 430 & & 520 & $100.0 \%$ \\
\hline Total events & 73 & & 93 & & \\
\hline
\end{tabular}

Odds Ratio Random, 95\% C $0.49[0.29,0.81]$ $1.56[0.99,2.46]$ $0.88[0.28,2.74]$

Test for overall effect: $Z=0.23(P=0.82)$

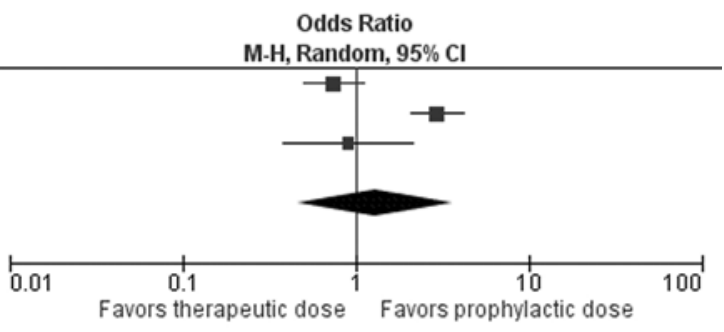

C

\begin{tabular}{|c|c|c|c|c|c|c|c|c|c|}
\hline \multirow[b]{2}{*}{ Study or Subgroup } & \multicolumn{2}{|c|}{ Therapeutic dose } & \multicolumn{2}{|c|}{ Prophylactic dose } & \multirow[b]{2}{*}{ Weight } & \multirow{2}{*}{$\begin{array}{c}\text { Odds Ratio } \\
\text { M-H, Random, } 95 \% \mathrm{Cl}\end{array}$} & \multirow{2}{*}{\multicolumn{3}{|c|}{$\begin{array}{c}\text { Odds Ratio } \\
\text { M-H, Random, } 95 \% \mathrm{Cl}\end{array}$}} \\
\hline & Events & Total & Events & Total & & & & & \\
\hline Bruder $2017^{16}$ & 53 & 197 & 63 & 197 & $49.1 \%$ & $0.78[0.51,1.21]$ & $\rightarrow$ & & \\
\hline Kole $2021^{19}$ & 22 & 233 & 59 & 323 & $45.4 \%$ & $0.47[0.28,0.79]$ & & & \\
\hline Simard $2013^{15}$ & 0 & 43 & 9 & 43 & $5.5 \%$ & $0.04[0.00,0.74]$ & $\leftarrow$ & & \\
\hline Total $(95 \% \mathrm{CI})$ & & 473 & & 563 & $100.0 \%$ & $0.53[0.26,1.07]$ & & & \\
\hline Total events & 75 & & 131 & & & & & & \\
\hline $\begin{array}{l}\text { Heterogeneity: } \mathrm{Tau}^{2} \\
\text { Test for overall effect }\end{array}$ & $\begin{array}{l}0.22 ; \mathrm{Chi}^{2} \\
\mathrm{Z}=1.77(\mathrm{P}\end{array}$ & $\begin{array}{l}76, d f= \\
08)\end{array}$ & $2(P=0.06)$ & $=65 \%$ & & & Favors therapeutic dose & $\begin{array}{c}10 \\
\text { Favors prophylactic dose }\end{array}$ & 100 \\
\hline
\end{tabular}

D

\begin{tabular}{|c|c|c|c|c|c|}
\hline \multirow[b]{2}{*}{ Study or Subgroup } & \multicolumn{2}{|c|}{ Therapeutic dose } & \multicolumn{2}{|c|}{ Prophylactic dose } & \multirow[b]{2}{*}{ Weight } \\
\hline & Events & Total & Events & Total & \\
\hline Simard $2013^{15}$ & 4 & 43 & 20 & 43 & $44.9 \%$ \\
\hline Kole $2021^{19}$ & 41 & 233 & 76 & 323 & $55.1 \%$ \\
\hline Total $(95 \% \mathrm{Cl})$ & & 276 & & 366 & $100.0 \%$ \\
\hline Total events & 45 & & 96 & & \\
\hline $\begin{array}{l}\text { Heterogeneity: Tau } \\
\text { Test for overall effe }\end{array}$ & $\begin{array}{l}1.37 ; \mathrm{Chi}^{2} \\
\mathrm{z}=1.31(\mathrm{P}\end{array}$ & $\begin{array}{l}59, \mathrm{df}= \\
19)\end{array}$ & $1(P=0.00$ & $=87 \%$ & \\
\hline
\end{tabular}

Odds Ratio , Random, 95\% C $0.12[0.04,0.39]$ $0.69[0.45,1.06]$

$0.31[0.06,1.77]$

FIG. 2. Forest plots showing efficacy outcomes between the therapeutic and prophylactic dose groups. A: Pooled estimates of angiographic vasospasm. B: Pooled estimates of severe angiographic vasospasm. C: Pooled estimates of cerebral infarction. D: Pooled estimates of clinical deterioration caused by DCI. $\mathrm{M}-\mathrm{H}=$ Mantel-Haenszel. 


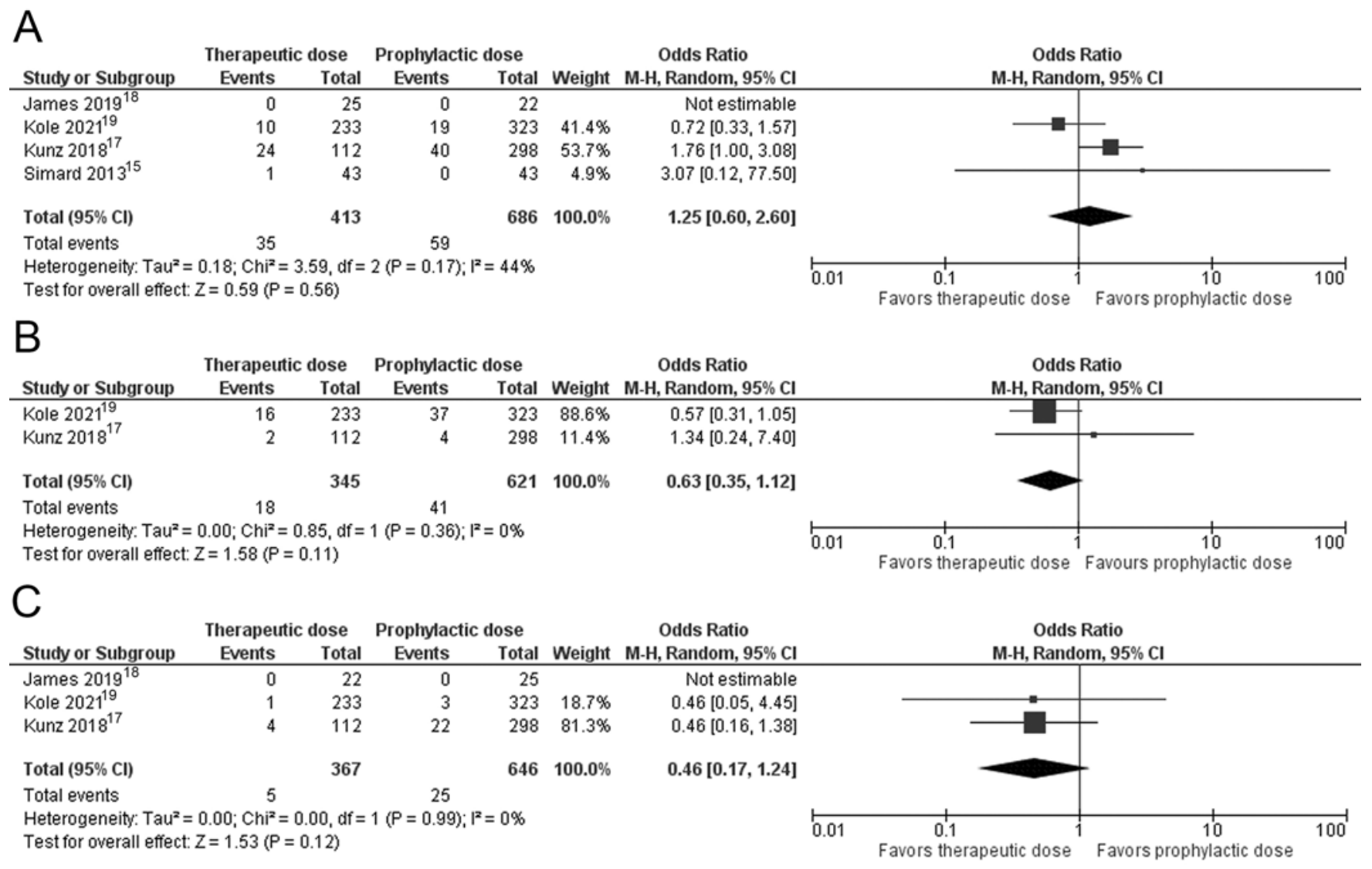

FIG. 3. Forest plots showing safety outcomes between the therapeutic and prophylactic dose groups. A: Pooled estimates of bleeding complications. B: Pooled estimates of DVT. C: Pooled estimates of HIT.

tistical significance with an OR of 0.63 (95\% CI $0.35-1.12$, $\mathrm{p}=0.11 ; \mathrm{I}^{2}=0 \%, \mathrm{p}=0.36$ ) (Fig. $3 \mathrm{~B}$ ). This finding shows that intravenous UFH is at least as effective as LMWH in preventing DVT.

\section{Heparin-Induced Thrombocytopenia}

Three studies assessed the occurrence of HIT, which was diagnosed as thrombocytopenia with both a positive heparin antibody test and serotonin release assay in the study by Kole et al., ${ }^{19}$ while James et al. and Kunz et al. did not describe their diagnostic method. ${ }^{17,18}$ These studies included 367 patients in the therapeutic group and 646 in the prophylactic group. Unexpectedly, the rate of HIT was lower in the therapeutic group although it did not reach statistical significance with an OR of 0.46 (95\% CI $0.17-$ $1.24, \mathrm{p}=0.12 ; \mathrm{I}^{2}=0 \%, \mathrm{p}=0.99$ ) (Fig. 3C).

\section{Sensitivity Analysis}

From the study by Bruder et al., we only included patients who had received intravenous UFH for $>48$ hours, while all patients from the studies by Simard et al. and Kole et al. were included because, in those 2 studies, treatment was administered for 12 to 16 days. ${ }^{15,16,19}$ There were 332 patients and 563 patients in the therapeutic and prophylactic groups, respectively. We found that the rate of cerebral infarction was significantly lower with an OR of $0.44\left(95 \%\right.$ CI $\left.0.25-0.79, \mathrm{p}=0.006 ; \mathrm{I}^{2}=31 \%, \mathrm{p}=0.24\right)$ (Fig. 4C). The therapeutic group still had a higher rate of angiographic vasospasm (Fig. 4A) but a lower rate of severe vasospasm (Fig. 4B), although these results were not statistically significant. This finding indicates that a longer administration time might have better efficacy.

\section{Certainty of Evidence}

The evidence was judged to be of very low to low certainty. Angiographic vasospasm, severe angiographic vasospasm, cerebral infarction, clinical deterioration caused by DCI, and bleeding complications had serious inconsistencies. The studies produced a wide range of point estimates and had an $\mathrm{I}^{2}$ value $>40 \%$. In the sensitivity analysis, all outcomes except cerebral infarction were judged to have serious imprecision because their pooled $95 \%$ CI overlapped 1.0. Table 3 gives a summary of the assessment.

\section{Discussion}

Our analysis found that administration of intravenous UFH for more than 48 hours after an aneurysm-securing procedure is significantly related to a lower rate of cerebral infarction (OR 0.44, 95\% CI 0.25-0.79). This difference was also observed when patients who received the treatment for less than 48 hours were included, although the difference is no longer significant. The type and method 


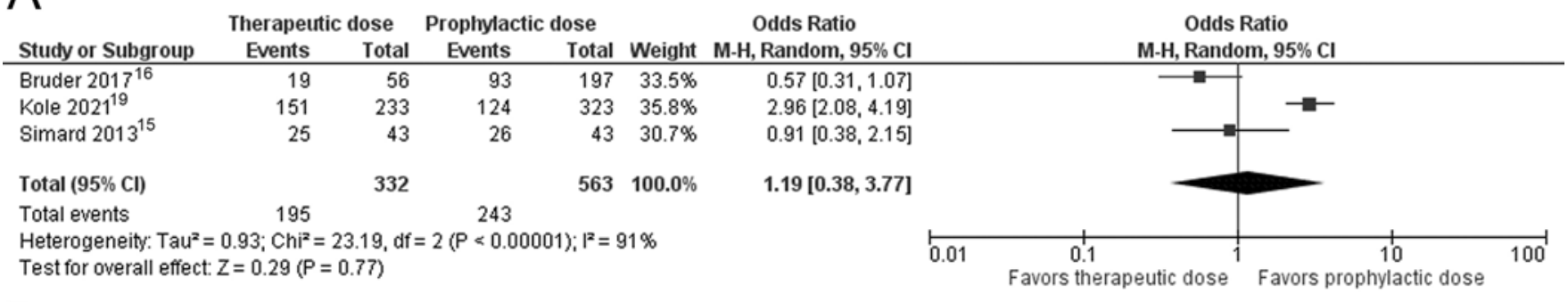

B

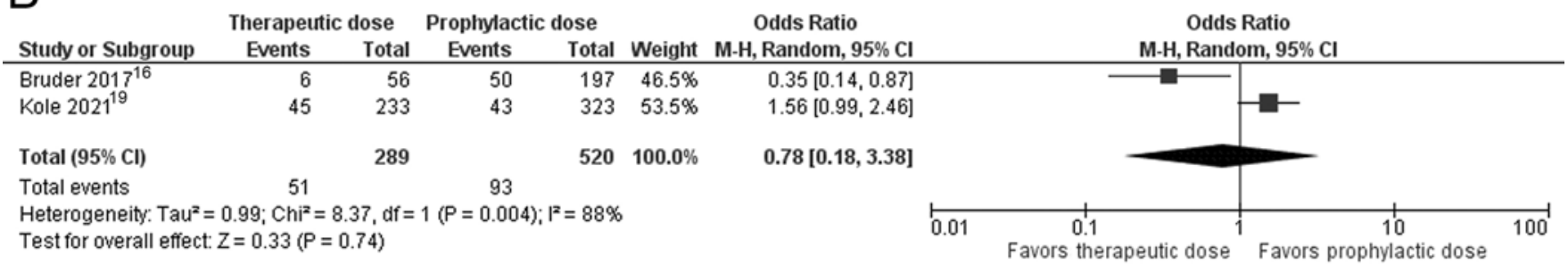

C

\begin{tabular}{|c|c|c|c|c|c|c|c|c|c|c|}
\hline \multirow[b]{2}{*}{ Study or Subgroup } & \multicolumn{2}{|c|}{ Therapeutic dose } & \multicolumn{2}{|c|}{ Prophylactic dose } & \multirow[b]{2}{*}{ Weight } & \multirow{2}{*}{\multicolumn{2}{|c|}{$\begin{array}{c}\text { Odds Ratio } \\
\text { M-H, Random, } 95 \% \mathrm{Cl}\end{array}$}} & \multirow{2}{*}{\multicolumn{2}{|c|}{$\begin{array}{c}\text { Odds Ratio } \\
\text { M-H, Random, } 95 \% \mathrm{Cl}\end{array}$}} & \\
\hline & Events & Total & Events & Total & & & & & & \\
\hline Bruder $2017^{16}$ & 11 & 56 & 63 & 197 & $39.7 \%$ & $0.52[0.25,1.07]$ & & & & \\
\hline Kole $2021^{19}$ & 22 & 233 & 59 & 323 & $56.4 \%$ & $0.47[0.28,0.79]$ & & & & \\
\hline Simard $2013^{15}$ & 0 & 43 & 9 & 43 & $3.9 \%$ & $0.04[0.00,0.74]$ & $\longleftarrow$ & & & \\
\hline Total $(95 \% \mathrm{Cl})$ & & 332 & & 563 & $100.0 \%$ & $0.44[0.25,0.79]$ & & & & \\
\hline Total events & 33 & & 131 & & & & & & & \\
\hline $\begin{array}{l}\text { Heterogeneity. Tau² } \\
\text { Test for overall effect }\end{array}$ & $\begin{array}{l}0.08 ; \mathrm{Chi}^{2}= \\
\mathrm{Z}=2.75(\mathrm{P}\end{array}$ & $\begin{array}{l}88, \mathrm{df}= \\
.006)\end{array}$ & $2(P=0.24)$ & $=31 \%$ & & & 0.01 & 0.1 s therapeutic dose & $\begin{array}{r}10 \\
\text { Favors prophylac }\end{array}$ & 100 \\
\hline
\end{tabular}

FIG. 4. Forest plots showing sensitivity analysis with patients who received intravenous heparin for $>48$ hours. A: Pooled estimates of angiographic vasospasm. B: Pooled estimates of severe angiographic vasospasm. C: Pooled estimates of cerebral infarction.

of heparin administration seem to have a significant effect on efficacy. Two previous randomized controlled trials with subcutaneous LMWH found conflicting results on the benefit of the intervention. ${ }^{20,21} \mathrm{UFH}$ is thought to have a higher binding capability to endothelial cells than LMWH, increasing its anti-inflammatory effect. ${ }^{22,23}$ All the studies included in this analysis administered intravenous UFH with varying protocols. The Maryland LDIVH protocol used in the studies by Simard et al., James et al., and Kole et al. calls for administration of intravenous heparin for 12 to 16 days after onset. ${ }^{15,18,19}$ Evaluation by aPTT was done twice daily, and if the value exceeded 45 seconds within the first 3 days, the infusion was decreased by one step for 12 hours before resuming the higher level. Bruder et al. administered heparin in a different manner in which they used an aPTT target of 60 seconds as a treatment goal for up to 7 days. ${ }^{16}$ Kunz et al. used an aPTT target of 50 to 60 seconds and 40 seconds each for the intervention and control group, respectively. ${ }^{17}$ From our sensitivity analysis, we found that the benefit was significant only when the patients who received heparin for $>48$ hours were included in the analysis. Infarction could occur within 24 hours to 6 days after the onset of aSAH. ${ }^{24}$ Therefore, administering $\mathrm{UFH}$ for a shorter duration might not prevent those infarctions that occurred after 48 hours. This finding shows that the beneficial effect of heparin is possibly affected by its type and duration of administration.
Cerebral infarction is an established prognostic factor for poor outcomes. ${ }^{25}$ Regarding clinical deterioration due to DCI, patients in the therapeutic group did have a lower occurrence, but the difference was not significant. However, aside from clinical deterioration caused by DCI, we were not planning to perform a meta-analysis on the functional and cognitive outcomes because of the expected heterogeneity of the parameters used. The study by James et al. ${ }^{18}$ evaluated outcomes by using the Montreal Cognitive Assessment (MoCA; https://www.mocatest.org/\#) and modified Rankin Scale (mRS) scores. They found that patients who received a therapeutic dose of heparin had a better mean MoCA score (26.4 vs 22.7, p = 0.013). In their study, $32 \%$ of patients in the prophylactic group had serious cognitive impairment compared with $0 \%$ in the therapeutic group $(p=0.008)$. Linear regression demonstrated that administration of the therapeutic dose of heparin for 12 to 14 days was associated with a positive influence on MoCA scores $(\beta=3.68, p=0.019)$. However, there was no statistically significant difference in mRS scores. ${ }^{18}$ Bruder et al. also evaluated clinical outcomes using the mRS, dividing them into favorable outcomes (mRS scores $0-2$ ) and unfavorable outcomes (mRS scores 3-6). The therapeutic group had a slightly better outcome, but the difference was not statistically significant $(69.0 \%$ vs $64.5 \%, \mathrm{p}=$ 0.57). ${ }^{16}$ Both studies were nonrandomized; hence, it was hard to control confounding bias. To address this issue, a 


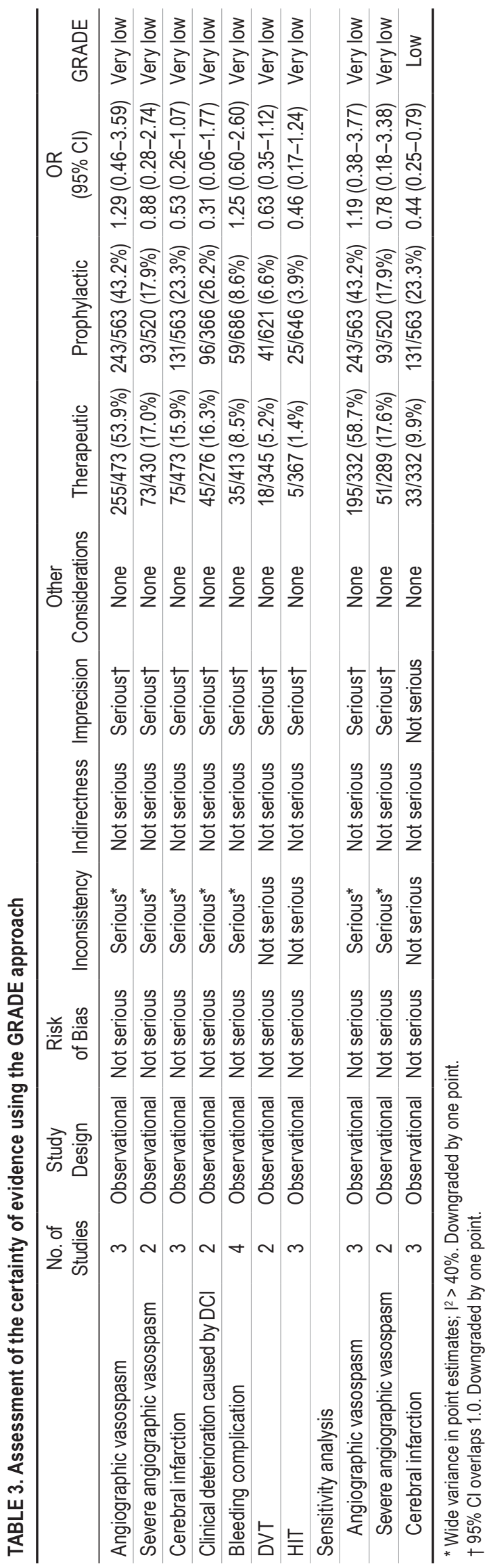

larger randomized trial would be needed to provide a definitive result.

Our analysis did not find a significant difference in the rate of angiographic vasospasm between the two groups. Interestingly, as mentioned before, cerebral infarction was found to be significantly lower in the therapeutic group. It has been agreed that angiographic vasospasm is a predictor for infarction, but the causality is still debated. Several studies have found that DCI could occur in the absence of angiographic vasospasm. ${ }^{26-28}$ Additional suggested mechanisms include microthromboembolism, microvascular constriction, neuroinflammation, blood-brain barrier disruption, cortical spreading ischemia, and impaired cerebral autoregulation. ${ }^{3,4}$ Heparin has been found to have the ability to bind with proinflammatory molecules and inflammation mediators. Moreover, it can reduce leukocyte extravasation in the central nervous system..$^{11}$ Therefore, heparin has the ability to reduce neuroinflammation. Studies have also found that heparin can reduce blood-brain barrier dysfunction and the resulting cerebral edema in traumatic brain injury, ischemic stroke, intracerebral hemorrhage, and meningitis. However, its effect on cerebral edema in patients with aSAH has not been studied. ${ }^{12}$ One mediator of microvascular constriction is endothelin-1 (ET-1). Heparin also can decrease the transcription of ET-1 and ET-1 promoters. ${ }^{29,30}$ Interestingly, one large randomized controlled trial evaluating the use of clazosentan, an ET-1 receptor antagonist, failed to find significant improvement in morbidity, mortality, or functional outcome. ${ }^{8}$ This result is replicated in our analysis in which heparin did not produce a significant difference in vasospasm incidence. This raises the possibility that heparin exerts its beneficial effect through a mechanism other than vasospasm prevention.

Our analysis found that patients in the intervention group had a higher rate of bleeding complications, although the difference was not significant. Heparin was not found to increase the occurrence of thrombocytopenia. In addition, our study found that intravenous UFH is at least as effective as subcutaneous UFH or LMWH in preventing DVT. These findings show that administration of intravenous heparin after an aneurysm-securing procedure is safe. Currently, a phase II clinical trial of intravenous heparin usage in aSAH is ongoing (registration no. NCT02501434, ClinicalTrials.gov). That trial started in April 2016 and planned to include 88 patients with a projected completion date of January 2024. No papers have described the recruitment number for this trial. One previous retrospective study by the principal investigator of that trial evaluated 47 patients over a 5-year period, resulting in an average of 9.4 enrolled patients each year. ${ }^{18}$ However, this trial is currently being conducted at several centers, which should increase the enrollment number. Another possible reason for the lengthy study plan is anticipation of the difficulty of obtaining patient consent, as it is logical for patients to think that administering heparin, an anticoagulant, is counterintuitive. The result of our analysis should support the ongoing trial by giving more assurance about the beneficial effect and good safety profile of intravenous heparin. 


\section{Limitations}

Some limitations need to be addressed. Regarding the review process, initially we did not plan on having a sensitivity analysis; however, we found an unexpected issue that necessitated the use of such analysis. We believe that this does not impact the validity of our review. Another limitation is the lack of randomized controlled studies in our analysis, which introduced potential bias. This is especially evident in confounding bias arising from the difference in patient baseline characteristics. In our analysis, the intervention group in the study by Kole et al. had statistically significantly worse prognostic factors. ${ }^{19}$ The authors adjusted for this difference using inverse probability of treatment weight analysis and found significant beneficial effects on cerebral infarction and neurological outcomes. However, since other studies in our analysis did not provide adjusted data, we decided to include only unadjusted data. Despite this difference, our analysis still showed a reduction in the rate of cerebral infarction and a good safety profile. Another concern is that the included studies did not use a uniform definition for outcomes. Regarding infarction, this opens the possibility that some of the reported cases were iatrogenic. Lack of standardized protocol for UFH administration also makes the comparison between studies difficult and poses a significant hurdle in implementing UFH as a treatment for aSAH.

\section{Conclusions}

Administration of intravenous UFH for more than 48 hours after an aneurysm-securing procedure reduced the rate of cerebral infarction. We found no significant benefits on angiographic vasospasm and clinical deterioration caused by DCI. Regarding its safety, intravenous UFH was not significantly associated with a higher rate of bleeding and HIT and was at least as good as DVT prophylaxis as subcutaneous UFH or LMWH. Despite the observational nature of the included studies and their inherent weakness and flaws, our analysis should support the aforementioned ongoing randomized controlled trial.

\section{References}

1. Al-Khindi T, Macdonald RL, Schweizer TA. Cognitive and functional outcome after aneurysmal subarachnoid hemorrhage. Stroke. 2010;41(8):e519-e536.

2. Macdonald RL, Schweizer TA. Spontaneous subarachnoid haemorrhage. Lancet. 2017;389(10069):655-666.

3. Budohoski KP, Guilfoyle M, Helmy A, et al. The pathophysiology and treatment of delayed cerebral ischaemia following subarachnoid haemorrhage. J Neurol Neurosurg Psychiatry. 2014;85(12):1343-1353.

4. Geraghty JR, Testai FD. Delayed cerebral ischemia after subarachnoid hemorrhage: beyond vasospasm and towards a multifactorial pathophysiology. Curr Atheroscler Rep. 2017; 19(12):50.

5. Miller BA, Turan N, Chau M, Pradilla G. Inflammation, vasospasm, and brain injury after subarachnoid hemorrhage. Biomed Res Int. 2014;2014:384342.

6. Dorhout Mees SM, Rinkel GJE, Feigin VL, et al. Calcium antagonists for aneurysmal subarachnoid haemorrhage. Cochrane Database Syst Rev. 2007;(3):CD000277.

7. Liu GJ, Luo J, Zhang LP, et al. Meta-analysis of the effectiveness and safety of prophylactic use of nimodipine in patients with an aneurysmal subarachnoid haemorrhage. CNS Neurol Disord Drug Targets. 2011;10(7):834-844.

8. Macdonald RL, Higashida RT, Keller E, et al. Clazosentan, an endothelin receptor antagonist, in patients with aneurysmal subarachnoid haemorrhage undergoing surgical clipping: a randomised, double-blind, placebo-controlled phase 3 trial (CONSCIOUS-2). Lancet Neurol. 2011;10(7):618-625.

9. De Oliveria Manoel AL, Loch Macdonald R. Neuroinflammation as a target for intervention in subarachnoid hemorrhage. Front Neurol. 2018;9:292.

10. Vergouwen MDI, Meijers JC, Geskus RB, et al. Biologic effects of simvastatin in patients with aneurysmal subarachnoid hemorrhage: a double-blind, placebo-controlled randomized trial. J Cereb Blood Flow Metab. 2009;29(8):1444-1453.

11. Hayman EG, Patel AP, James RF, Simard JM. Heparin and heparin-derivatives in post-subarachnoid hemorrhage brain injury: a multimodal therapy for a multimodal disease. Molecules. 2017;22(5):724.

12. Khattar NK, James RF. Heparin: the silver bullet of aneurysmal subarachnoid hemorrhage? Front Neurol. 2018;9:97.

13. Stutzmann JM, Mary V, Wahl F, Grosjean-Piot O, Uzan A, Pratt J. Neuroprotective profile of enoxaparin, a low molecular weight heparin, in in vivo models of cerebral ischemia or traumatic brain injury in rats: a review. CNS Drug Rev. 2002;8(1):1-30.

14. Vergouwen MDI, Vermeulen M, van Gijn J, et al. Definition of delayed cerebral ischemia after aneurysmal subarachnoid hemorrhage as an outcome event in clinical trials and observational studies: proposal of a multidisciplinary research group. Stroke. 2010;41(10):2391-2395.

15. Simard JM, Aldrich EF, Schreibman D, James RF, Polifka A, Beaty N. Low-dose intravenous heparin infusion in patients with aneurysmal subarachnoid hemorrhage: a preliminary assessment. J Neurosurg. 2013;119(6):1611-1619.

16. Bruder M, Won SY, Kashefiolasl S, et al. Effect of heparin on secondary brain injury in patients with subarachnoid hemorrhage: an additional ' $\mathrm{H}$ ' therapy in vasospasm treatment. $J$ Neurointerv Surg. 2017;9(7):659-663.

17. Kunz M, Siller S, Nell C, et al. Low-dose versus therapeutic range intravenous unfractionated heparin prophylaxis in the treatment of patients with severe aneurysmal subarachnoid hemorrhage after aneurysm occlusion. World Neurosurg. 2018;117:e705-e711.

18. James RF, Khattar NK, Aljuboori ZS, et al. Continuous infusion of low-dose unfractionated heparin after aneurysmal subarachnoid hemorrhage: a preliminary study of cognitive outcomes. J Neurosurg. 2019;130(5):1460-1467.

19. Kole MJ, Wessell AP, Ugiliweneza B, et al. Low-dose intravenous heparin infusion after aneurysmal subarachnoid hemorrhage is associated with decreased risk of delayed neurological deficit and cerebral infarction. Neurosurgery. 2021; 88(3):523-530.

20. Siironen J, Juvela S, Varis J, et al. No effect of enoxaparin on outcome of aneurysmal subarachnoid hemorrhage: a randomized, double-blind, placebo-controlled clinical trial. $J$ Neurosurg. 2003;99(6):953-959.

21. Wurm G, Tomancok B, Nussbaumer K, Adelwöhrer C, Holl $\mathrm{K}$. Reduction of ischemic sequelae following spontaneous subarachnoid hemorrhage: a double-blind, randomized comparison of enoxaparin versus placebo. Clin Neurol Neurosurg. 2004;106(2):97-103.

22. Young E, Venner T, Ribau J, Shaughnessy S, Hirsh J, Podor TJ. The binding of unfractionated heparin and low molecular weight heparin to thrombin-activated human endothelial cells. Thromb Res. 1999;96(5):373-381.

23. Simard JM, Schreibman D, Aldrich EF, et al. Unfractionated heparin: multitargeted therapy for delayed neurological deficits induced by subarachnoid hemorrhage. Neurocrit Care. 2010;13(3):439-449.

24. Ayling OGS, Ibrahim GM, Alotaibi NM, Gooderham PA, 
Macdonald RL. Dissociation of early and delayed cerebral infarction after aneurysmal subarachnoid hemorrhage. Stroke. 2016;47(12):2945-2951.

25. Vergouwen MDI, Ilodigwe D, Macdonald RL. Cerebral infarction after subarachnoid hemorrhage contributes to poor outcome by vasospasm-dependent and -independent effects. Stroke. 2011;42(4):924-929.

26. Brown RJ, Kumar A, Dhar R, Sampson TR, Diringer MN. The relationship between delayed infarcts and angiographic vasospasm after aneurysmal subarachnoid hemorrhage. $\mathrm{Neu}$ rosurgery. 2013;72(5):702-708.

27. Crowley RW, Medel R, Dumont AS, et al. Angiographic vasospasm is strongly correlated with cerebral infarction after subarachnoid hemorrhage. Stroke. 2011;42(4):919-923.

28. Dhar R, Diringer MN. Relationship between angiographic vasospasm, cerebral blood flow, and cerebral infarction after subarachnoid hemorrhage. Acta Neurochir Suppl. 2015;120: 161-165.

29. Kuwahara-Watanabe K, Hidai C, Ikeda H, et al. Heparin regulates transcription of endothelin-1 gene in endothelial cells. J Vasc Res. 2005;42(3):183-189.

30. Yokokawa K, Mandal AK, Kohno M, et al. Heparin suppresses endothelin-1 action and production in spontaneously hypertensive rats. Am J Physiol. 1992;263(5 Pt 2):R1035R1041.

\section{Disclosures}

The authors report no conflict of interest concerning the materials or methods used in this study or the findings specified in this paper.

\section{Author Contributions}

Conception and design: Lukito. Acquisition of data: Lukito, Lie. Analysis and interpretation of data: Lukito, Lie, Helsa. Drafting the article: Lukito, Lie, Helsa. Critically revising the article: July. Reviewed submitted version of manuscript: Lukito, July. Approved the final version of the manuscript on behalf of all authors: Lukito. Statistical analysis: Lukito, Lie. Study supervision: July.

\section{Supplemental Information}

Previous Presentations

Portions of this paper were presented at the 24th Annual Scientific Meeting of Indonesian Neurosurgical Society (PIT PERSPEBSI XXIV), Aceh, Indonesia, July 24, 2021.

\section{Correspondence}

Patrick P. Lukito: Universitas Pelita Harapan, Tangerang, Banten, Indonesia.lukito_patrick@yahoo.co.id. 